\title{
Esterilização em gatas mediante salpingectomia parcial (incluindo prenhes) versus ovariossalpingohisterectomia
}

\author{
Sterilization in queens by partial salpingectomy (including pregnant) versus ovariohysterectomy
}

\author{
Amanda Camilo Silva ${ }^{\mathrm{I}}$ Camila Elana Santana e Silva"I Emília Miranda Peluso ${ }^{\mathrm{II}}$ \\ Eduardo Alberto Tudury ${ }^{\mathrm{III}}$
}

\section{RESUMO}

A ligadura ou laqueadura das tubas uterinas é uma forma de contracepção bastante utilizada na medicina humana. Ela consiste na oclusão mecânica e/ou ressecção parcial desta estrutura (salpingectomia parcial). Nesta pesquisa, procurou-se utilizar uma técnica de contracepção definitiva em gatas prenhes, que não comprometesse a gestação em curso, o parto, nem os conceptos, tendo em vista a frequência da visualização de útero gravídico nas campanhas de castração. Avaliou-se também o ganho de peso e mudanças de comportamento das gatas submetidas à salpingectomia parcial, comparando-as com as submetidas à ovariossalpingohisterectomia (OSH), além de comparar o tempo cirúrgico entre os dois procedimentos. Para tal, foram utilizadas 40 gatas, distribuídas em dois grupos de 20 animais, GA: salpingectomia parcial, e GB: OSH; sendo o primeiro subdividido em GA1 contendo 10 gatas prenhes e GA2 contendo 10 não prenhes. Todos os animais foram reavaliados com sete, 60, 180 e 365 dias. O tempo cirúrgico apresentou diferença estatisticamente significativa entre os grupos, sendo o GB mais demorado. Nas gatas do grupo GA1, o curso gestacional e o parto foram normais. No total, foram visualizadas 51 vesículas embrionárias, nasceram 49 fetos vivos e ocorreram duas reabsorções fetais. Dentre os 49 fetos, sete $(14,28 \%)$ apresentaram a anormalidade genu recurvatum. Quanto à fertilidade, os animais do grupo A que ciclaram e copularam não engravidaram. Apenas o grupo $B$ apresentou aumento de peso médio (20,34\%) estatisticamente significativo. Com relação aos parâmetros comportamentais, o grupo $B$ apresentou maiores alterações quanto ao aumento na ingestão de alimentos, aumento da letargia e diminuição do período de vigília. Concluiu-se que a técnica de salpingectomia parcial realizada em gatas é um método contraceptivo $100 \%$ eficaz, de rápida execução, que pode ser empregado durante a identificação transcirúrgica do estado de prenhez, com mínimo efeito prejudicial aos conceptos e sem alterações significativas de conduta e ganho de peso, mas com características indesejáveis quanto à aceitação dos proprietários.

Palavras-chave: contracepção, gestação, tuba uterina, ovariossalpingohisterectomia.

\section{ABSTRACT}

The tubal occlusion is a form of contraception used in human medicine, which consists of mechanical occlusion and / or partial resection of this structure (partial salpingectomy). In this study, we tried to use a technique for definitive contraception in pregnant queens, that would not compromise the current pregnancy, the birth of kittens, and the concepts, considering the frequency of the visualization of gravid uterus in castration campaigns. Another objective was evaluate weight gain and changes in behavior of queens submitted to partial salpingectomy, comparing them with those submitted to ovariohysterectomy (OSH), and to compare the time between both surgical procedures. Were used 40 queens, divided into two groups of 20 animals, GA: Partial salpingectomy, and GB: OSH. The group GA, was divided in GA1, containing 10 queens pregnant and GA2 containing 10 non-pregnant. All animals were assessed with seven, 60, 180 and 365 days. The surgical times showed statistically significant difference between the groups, with GB slower. The gestacional course and birth of kittens were normal in queens of group GA1. A total of 51 embryonic vesicles were visualized, 49 born live fetuses, and occurring two fetals resorptions. Among the 49 fetuses, seven (14.28\%) presented genu recurvatum. With respect to fertility, the animals in group $A$ that cycled and mated, did not get pregnant. Only the Group B showed average weight increase (20.34\%) statistically significant. Regarding

'Programa de Pós-Graduação em Ciência Veterinária da Universidade Federal Rural de Pernambuco (UFRPE), Rua Dom Manoel de Medeiros, s/n, 52171-900, Recife, PE, Brasil. E-mail: amandacamilovet@yahoo.com.br. Autor para correspondência.

${ }^{\text {IIC } C u r s o ~ d e ~ M e d i c i n a ~ V e t e r i n a ́ r i a ~ d a ~ U F R P E, ~ R e c i f e, ~ P E, ~ B r a s i l . ~}$

IIIDepartamento de Medicina Veterinária da UFRPE, Recife, PE, Brasil. 
the behavioral parameters, the group B showed increase in food intake, increase of the lethargy, and decreased in waking period. It is concluded that the technique of partial salpingectomy performed in queens is a $100 \%$ effective contraceptive method of rapid execution, which can be employed during the transoperative identification of pregnancy, with minimal adverse effects on fetuses, no significant changes of behavior neither weight gain, but with undesirable characteristics on the acceptance of owners.

Key words: contraception, pregnancy, oviduct, ovariohysterectomy.

\section{INTRODUÇÃO}

As opções disponíveis para prevenção da gestação em gatas e cadelas continuam limitadas, destacando-se a OSH e a contracepção hormonal (CONCANNON \& MEYERS-WALLEN, 1991; JOHNSON, 2006a). Embora a OSH seja considerada um procedimento tecnicamente simples, podem ocorrer complicações no trans e pós-cirúrgico imediato, mediato e tardio, destacando-se as hemorragias (SANTOS et al., 2009). Outras possíveis complicações são: ligadura ou trauma ao ureter, incontinência urinária, formação de tratos fistulosos e granulomas, síndrome do ovário remanescente, problemas relacionados à celiotomia, piometra de coto, obstruções intestinais (SANTOS et al., 2009), ganho de peso (DORN \& SWIST, 1977; STONE, 2002) e provável mudança de comportamento (SOARES \& SILVA, 1998). A contracepção hormonal apresenta bons resultados, mas pode causar efeitos colaterais como aumento de peso, hiperplasia ou neoplasia mamária, piometra, diabete melito e supressão adrenal (CONCANNON \& MEYERS-WALLEN, 1991; ESMERALDO et al., 2007; ENDO et al., 2009).

A ligadura ou laqueadura das tubas uterinas é um método de contracepção cirúrgica permanente (MODOTTE, et al., 2004), com possibilidade de reversão, bastante utilizado na medicina humana (REGGIANI et al., 2000; FIGUERÊDO et al., 2006). O citado procedimento apresenta taxa de falha baixa $(0,1$ a $0,8 \%$ ) e não interfere na saúde do animal, tendo em vista que a função endócrina dos ovários é mantida, não interferindo na libido, nem resultando em efeitos colaterais a longo prazo (STEELE, 1995; WILSON, 1996).

Várias técnicas podem ser utilizadas na realização da ligadura das tubas uterinas, sejam elas por laparotomia, laparoscopia ou via vaginal (MODOTTE et al., 2004). A técnica mais conhecida é a Pomeroy, utilizada desde a década de 1930. A partir desta, muitas outras foram descritas envolvendo ligadura e ressecção parcial tubária (salpingectomia parcial), sendo algumas simples e outras mais complexas (WILSON, 1996).
Independente do procedimento utilizado, o princípio é sempre o mesmo - bloquear a passagem ascendente dos espermatozoides e descendente dos ovócitos, evitando-se, assim, a fecundação (MODOTTE et al., 2004). Para essa finalidade, podem ser utilizados fios de sutura, anéis plásticos, clipes de titânio, eletrocauterização, entre outros (WILSON, 1996).

Objetivou-se com este trabalho, utilizar uma técnica de contracepção definitiva em gatas prenhes, que não comprometesse a gestação em curso, o parto, nem os conceptos, tendo em vista que é comum em campanhas de esterilização a visualização de útero gravídico durante a abordagem à cavidade abdominal. Avaliou-se também o ganho de peso e as mudança de comportamento das gatas submetidas à salpingectomia parcial, comparando-as com as submetidas à OSH, além de comparar o tempo cirúrgico entre os dois procedimentos.

\section{MATERIAL E MÉTODOS}

Após aprovação pela CEUA/UFRPE e liberação de licença no 026/2009, foram utilizados 40 animais da espécie felina (Felis catus), fêmeas, entre um e cinco anos de idade, trazidos ao Hospital Veterinário da UFRPE para realização de contracepção cirúrgica. Estes foram distribuídos em dois grupos. $\mathrm{O}$ GA composto por 20 gatas que foram submetidas ao procedimento cirúrgico de salpingectomia parcial; e o GB com 20 animais submetidos ao procedimento cirúrgico de OSH. O GA foi subdividido em GA1, contendo 10 gatas prenhes, e GA2, contendo 10 não prenhes.

Em cada paciente, foi realizada a resenha clínica, anamnese, exame físico e exames pré-cirúrgicos: hemograma, tempo de coagulação e tempo de sangria. Foram incluídos na pesquisa os animais que se encontravam em condição orgânica estável e apropriada para anestesia e cirurgia, e cujos proprietários concordaram por escrito com a realização do procedimento.

No protocolo anestésico, foi utilizado sulfato de atropina $^{\mathrm{a}}\left(0,044 \mathrm{mg} \mathrm{kg}^{-1}\right)$, cloridrato de xilazina ${ }^{\mathrm{b}}(1 \mathrm{mg}$ $\left.\mathrm{kg}^{-1}\right)$ e cloridrato de tramadol ${ }^{\mathrm{c}}\left(2 \mathrm{mg} \mathrm{kg}^{-1}\right)$ como préanestésicos, todos por via subcutânea; cloridrato de cetamina $^{\mathrm{d}}$ (10 $\mathrm{mg} \mathrm{kg}^{-1}$, via intramuscular) na indução e (2,5 a $5 \mathrm{mg} \mathrm{kg}^{-1}$ via intravenosa) na manutenção anestésica.

A abordagem à cavidade abdominal, para realização da salpingectomia parcial, ocorreu através da incisão de $3,0 \mathrm{~cm}$ na pele e subcutâneo, na linha média ventral, iniciando $3,0 \mathrm{~cm}$ caudalmente à cicatriz umbilical. Após a incisão da linha alba, teve-se acesso 
aos ovários e tubas uterinas. A seguir, foi realizada a técnica Parkland modificada, em que, após divulsão da tuba uterina na face lateral da bursa ovárica, realizou-se o pinçamento dessa estrutura com duas pinças hemostáticas Halsted-mosquito, afastadas aproximadamente de 0,5 a $1 \mathrm{~cm}$ uma da outra. Procedeu-se à exérese do segmento entre as duas pinças e à colocação de ligaduras com fio de náilon 5-0, substituindo cada uma das pinças hemostáticas (Figura 1), preservando a irrigação ovariana. Na celiorrafia, foi utilizado fio de náilon 3-0 em pontos de Sultan na linha alba; no subcutâneo, utilizou-se categute cromado 3-0 em padrão contínuo intradérmico em ziguezague; e, na pele, fio de náilon $n^{\circ}$ 3-0 em padrão isolado simples.

Para o grupo submetido à OSH, a técnica de abordagem e oclusão foi a mesma descrita anteriormente, sendo realizada ligadura circular em massa em cada pedículo ovariano com categute cromado 2-0, e ligadura transfixante do coto uterino com categute cromado 2-0.

Durante a realização do procedimento de salpingectomia parcial, foram registradas as dificuldades e complicações encontradas, assim como a quantidade de vesículas embrionárias observadas. Também se avaliou o tempo de execução de ambos os procedimentos (salpingectomia parcial e OSH), sempre realizados pelo mesmo cirurgião. O registro do tempo foi dividido da seguinte forma: T1 (início da cirurgia que correspondeu ao início da incisão até a abordagem ao ovário), T2 (início da OSH ou da salpingectomia parcial), T3 (fim da OSH ou da salpingectomia parcial) e T4 (fim da cirurgia que correspondeu à celiorrafia).

Após as cirurgias, os pacientes receberam anti-inflamatório oral, cetoprofeno ${ }^{\mathrm{e}}\left(1 \mathrm{mg} \mathrm{kg}^{-1}\right)$ a cada 24 horas, durante três dias. A antissepsia da ferida cirúrgica era realizada com clorexidina uma vez ao dia até a retirada dos pontos no oitavo dia.

Todos os animais foram reavaliados com sete, 60, 180 e 365 dias, momentos nos quais foram observados os seguintes parâmetros: manutenção da gestação, ocorrência de reabsorção fetal (na primeira reavaliação, com acompanhamento assistencial até o parto), fertilidade pós salpingectomia parcial, ganho de peso e mudanças de comportamento relacionadas às alterações do período de vigília, letargia e ingestão de alimentos, observada entre os animais dos grupos A e B. Foram realizados questionários com os proprietários, evidenciando sua observação quanto às mudanças comportamentais, e sua satisfação e aceitação quanto ao procedimento cirúrgico.

Para a variável ganho de peso, foi utilizado o teste estatístico não paramétrico para amostras dependentes de Wilcoxon, e, para a variável tempo de execução, foi utilizado o teste não paramétrico para amostras independentes de Mann-withney, ambos com nível de significância de 5\%. As análises estatísticas foram realizadas através do software estatístico Statistica (Copyrigtht ${ }^{\circledR}$ StatSoft) versão 5.5.

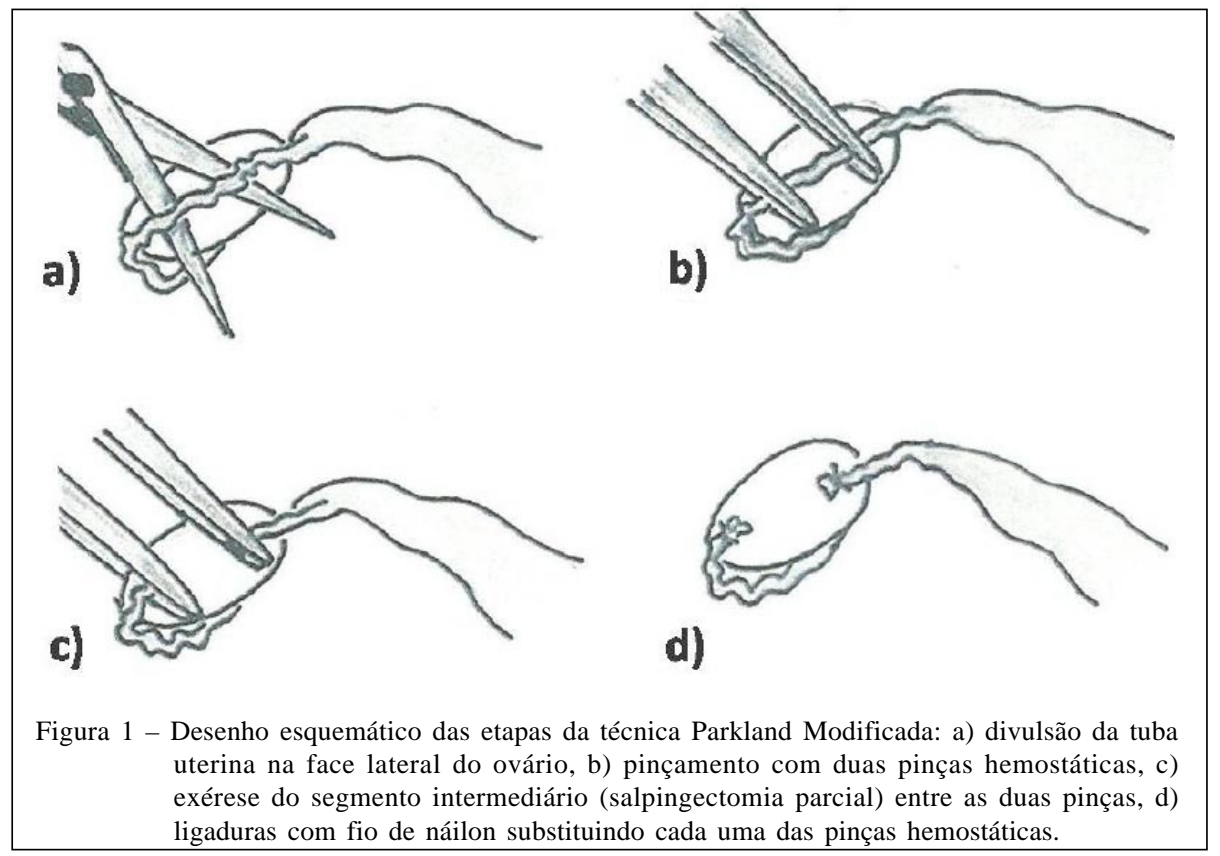

Ciência Rural, v.42, n.3, mar, 2012. 


\section{RESULTADOS E DISCUSSÃO}

O tempo médio da salpingectomia parcial, obtido entre T2 e T3, foi de 353 segundos (5 minutos e 53 segundos) com desvio padrão de 58,476, enquanto que o da OSH correspondeu a 448 segundos ( 7 minutos e 28 segundos), com desvio padrão de 84,609. O maior tempo cirúrgico da $\mathrm{OSH}$ ocorreu devido à realização de reforços nas ligaduras com intuito de coibir hemorragias dos pedículos ovarianos ou do coto uterino e, ainda, ocasionalmente, devido ao esvaziamento vesical transcirúrgico.

Com base no descrito acima, verificou-se diferença média de 1 minuto e 35 segundos entre as técnicas. Estatisticamente, obteve-se diferença significativa $(\mathrm{p}=0,000069)$, diante do teste de MannWithney. Dessa forma, vê-se que o tempo de execução da salpingectomia parcial é menor, proporcionando redução no que diz respeito ao tempo anestésico e à exposição das estruturas abdominais ao meio externo, além de, possivelmente, diminuir a possibilidade de contaminação.

Ainda com base nas observações transcirúrgicas, a realização do procedimento de salpingectomia parcial não apresentou nenhuma complicação. Em relação às dificuldades encontradas, foi observado que quanto menor a paciente, maior a dificuldade de visualização e divulsionamento das tubas uterinas, tendo em vista que se trata de uma estrutura estreita de até $1,5 \mathrm{~mm}$ nas gatas aqui analisadas, podendo chegar a $3 \mathrm{~mm}$ em cadelas, como descrito por EVANS \& CHRISTENSEN (1993).

Nas dez gatas gestantes submetidas à salpingectomia parcial, em diferentes períodos da prenhez, o curso gestacional foi normal, não sendo observadas complicações relacionadas com perda de fluidos vaginais e expulsão de embrião ou feto prematuro, ocorrendo o aumento natural do volume abdominal e do peso, compatível com o desenvolvimento dos conceptos. O procedimento também não proporcionou complicações relacionadas ao parto, não havendo episódios de distocia.

No total, foram visualizadas transcirurgicamente 51 vesículas embrionárias, entretanto, nasceram 49 fetos vivos, nenhum natimorto e nenhuma retenção fetal observada através de exame ultrassonográfico. Dessa forma, ocorreram dois (3,92\%) episódios de reabsorção fetal. A interrupção do desenvolvimento fetal é multifatorial, podendo ser causada por fatores endócrinos, nutricionais, traumáticos, infecciosos ou por anomalias (MOORE \& PERSAUD, 2004). A etiologia das duas reabsorções fetais está provavelmente associada a anomalias ou à utilização de fármacos, tendo em vista que a técnica empregada não parece ter proporcionado lesões fetais ou comprometimento da irrigação ovariana, considerando ainda que os animais apresentavam boas condições nutricionais e que processos infecciosos não estavam clinicamente presentes. JOHNSON (2006b) descreve que as anomalias do desenvolvimento em fetos não possuem causa identificável, especulando-se fatores ambientais, como exposição a teratógenos e aberrações cromossômicas.

Dentre os 49 fetos, sete $(14,28 \%)$ apresentaram a afecção denominada genu recurvatum, caracterizada pela hiperextensão das articulações tíbiofemuro-patelar e tíbio-társica, com dificuldade de flexão dos membros acometidos, tornando-os rígidos e afuncionais (RUDY, 1974). JONES et al. (2000) descrevem que a causa das anormalidades congênitas são geralmente desconhecidas. Diante do fato de os filhotes terem sido concebidos por duas gatas de origem genética diferente, não é possível atribuir que fatores hereditários sejam a causa das alterações obtidas. Acredita-se que as alterações estavam associadas a fatores ambientais, mais precisamente ao uso de fármacos, tendo em vista que ambos os animais foram submetidos ao mesmo protocolo anestésico e antiinflamatório, durante o período gestacional. A possibilidade viral não foi considerada, já que apenas animais saudáveis foram submetidos ao procedimento e que, no pós-operatório, tais pacientes não demonstravam sinais de infecção por esses agentes.

Com o intuito de minimizar a quantidade de quimioterápicos administrados nas gatas gestantes durante o pós-cirúrgico, já que os fármacos variam quanto a sua teratogenicidade (MOORE \& PERSAUD, 2004), utilizou-se apenas o cetoprofeno, não sendo observados sinais dolorosos. Entretanto, haja vista o estímulo doloroso associado ao procedimento, é importante ressaltar que melhor analgesia pode ser obtida através da associação entre anti-inflamatório não esteroide e opióde (CHIORATTO \& TUDURY, 2009).

Dentre todos os fármacos utilizados durante anestesia e pós-cirúrgico, nenhum destes apresentam efeitos teratogênicos cientificamente comprovados, e a dosagem foi utilizada de acordo com VIANA (2007). Logo, desperta a necessidade da realização de estudos mais específicos. O conhecimento de que certos fármacos podem perturbar o desenvolvimento pré-natal oferece a oportunidade para prevenir seu uso no período gestacional.

Quanto à fertilidade, das 20 gatas submetidas à salpingectomia parcial, 18 retornaram ao estro, 17 acasalaram e nenhuma apresentou nova 
gestação. Esses dados foram coletados após observações durante um período de 365 dias, o que mostra que a técnica empregada é um método contraceptivo 100\% eficaz, corroborando STEELE (1995) e WILSON (1996), quando citam que a eficácia desse método é muito alta. É relevante considerar que a probabilidade de ocorrer recanalização com posterior gestação, neste experimento, é praticamente nula, devido à opção pela exérese de segmento tubário e ligadura das extremidades remanescentes com fio não absorvível.

Após um ano de avaliação, quanto ao aumento de peso das gatas, foi observado que o procedimento que mais influenciou no ganho de peso foi a OSH (Figura 2), que resultou em aumento médio de $20,34 \%$, enquanto que a salpingectomia parcial resultou em aumento de 9,57\%. Esses achados estão de acordo com STONE (2002), quando sugere que os depósitos de gordura do corpo possuem receptores para hormônios esteroides específicos, de forma a facilitar ou a inibir a deposição de gordura de maneira regional em resposta à estes hormônios. Como o estradiol inibe a lípase lipoproteica nos adipócitos dos depósitos gordurosos resultando na não esterificação e deposição dos ácidos graxos circulantes, pode-se, consequentemente, compreender que um baixo nível de estradiol sistêmico após a OSH pode levar a uma deposição excessiva de gordura com consequente ganho de peso.

Estatisticamente, o teste Wilcoxon demonstrou que o aumento de peso das gatas submetidas à salpingectomia parcial foi considerado não significativo $(\mathrm{P}=0,130570)$, enquanto que o aumento de peso das gatas submetidas à OSH foi considerado extremamente significativo $(\mathrm{P}=0,000062)$.

Com base nos resultados obtidos em relação à avaliação comportamental respondida pelos proprietários dos animais operados, observou-se que $60 \%$ das gatas do grupo B passaram a comer mais, enquanto que $75 \%$ dos animais do grupo A não modificaram seu hábito alimentar. Essa modificação na dieta está de acordo com PIBOT et al. (2008), quando citam que os estrogênios podem suprimir o apetite, portanto, a remoção deles pela gonadectomia pode levar ao aumento da ingestão de alimentos, com consequente ganho de peso. Isso justificaria o rápido aumento de peso observado nos dois primeiros meses pós-cirúrgicos das gatas submetidas à OSH (Figura 02), pelas modificações endócrinas ainda não adaptadas.

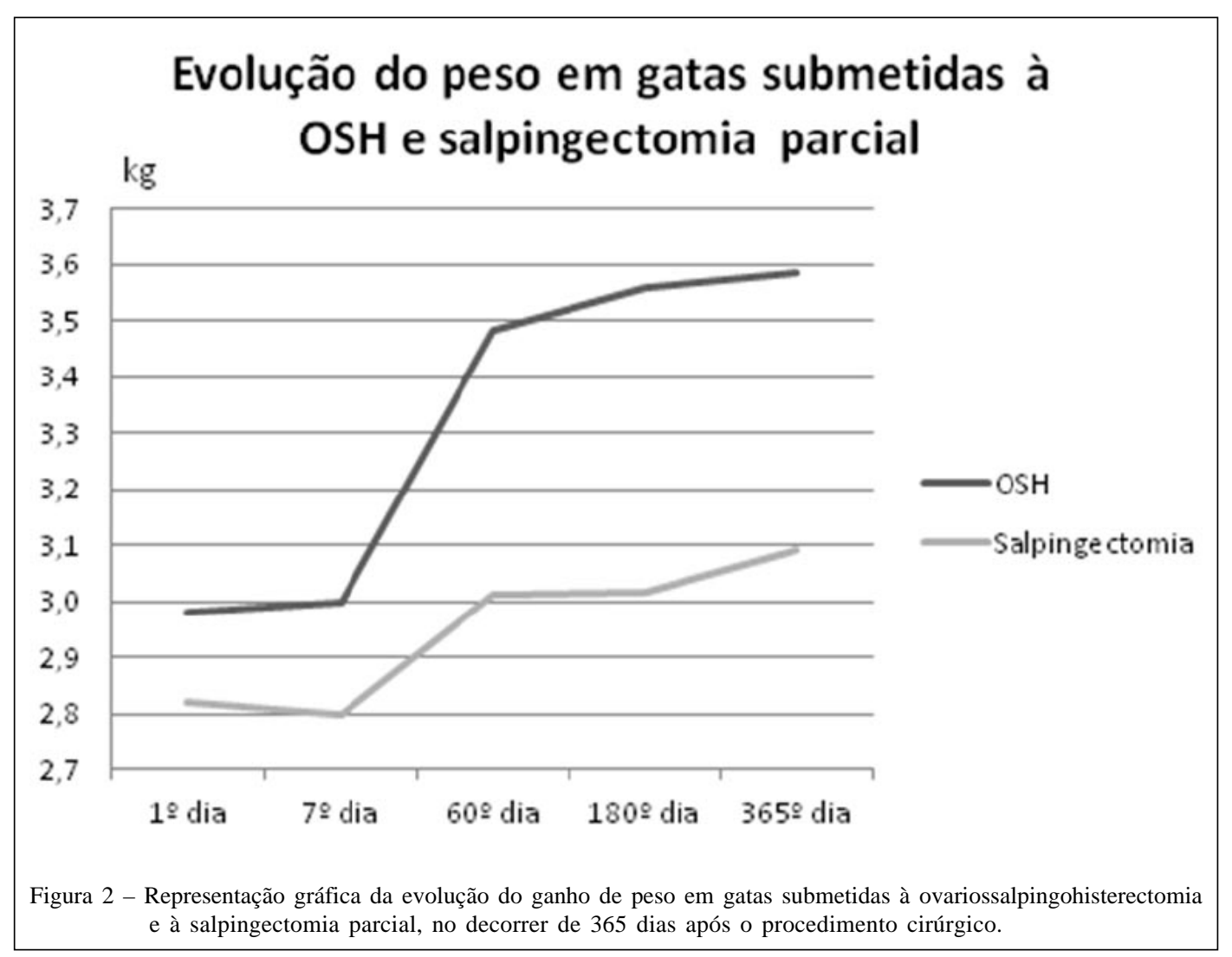

Ciência Rural, v.42, n.3, mar, 2012. 
Ainda quanto às diferenças comportamentais, observou-se que $65 \%$ dos animais do grupo B ficaram letárgicos após a realização da cirurgia, enquanto que $80 \%$ dos animais do grupo A permaneceram com seu comportamento inalterado. Ficou constatado também que $75 \%$ das gatas do grupo B estavam dormindo mais, enquanto $90 \%$ das gatas do grupo A não exibiram diferenças quanto ao período de vigília, concordando com PIBOT et al. (2008), quando descrevem que após a castração ocorre diminuição da atividade física.

Segundo SOARES \& SILVA (1998), os parâmetros comportamentais são de difícil avaliação, pois não se sabe até que ponto o próprio envelhecimento pode influenciar nessa característica. Entretanto, aqui fica evidente que tal parâmetro foi influenciado pelo procedimento realizado, tendo em vista que os animais de ambos os grupos tinham a mesma faixa etária, e que apenas os submetidos à OSH apresentaram mudanças comportamentais significativas em um curto espaço de tempo (365 dias).

Baseado nos parâmetros comportamentais analisados, observa-se que a salpingectomia parcial é uma técnica favorável, pois imputa o zelo pelo bemestar animal, considerando que os animais são seres sencientes. A citada técnica proporciona ainda boa qualidade de vida aos animais, respeitando a satisfação das suas necessidades físicas, mentais e naturais, conforme regulamenta a legislação hora vigente (resolução CFMV Nº 879, de 15 de fevereiro de 2008).

Um importante item analisado durante o experimento foi a satisfação do proprietário com relação ao procedimento cirúrgico, em que $80 \%$ dos proprietários das gatas gestantes consideraram a salpingectomia parcial um bom procedimento, pois evitou o aborto durante a abordagem cirúrgica ao mesmo tempo em que realizou uma forma de contracepção, não interferindo na gestação nem no parto. Os proprietários das gatas não gestantes consideraram o procedimento apropriado em $60 \%$ dos casos, tendo em vista que o método contraceptivo foi eficaz, mas o estro ainda existente foi considerado indesejável. A OSH, por sua vez, atingiu 100\% de satisfação dos proprietários, por ser um método contraceptivo eficaz que inibe o estro.

\section{CONCLUSÃO}

Conclui-se que a técnica de salpingectomia parcial descrita em gatas é um método contraceptivo $100 \%$ eficaz, de rápida execução, que pode ser empregado durante a identificação transcirúrgica do estado de prenhez, com mínimo efeito prejudicial aos conceptos e sem alterações significativas de conduta e ganho de peso, mas com características indesejáveis quanto à aceitação dos proprietários.

\section{FONTES DE AQUISIÇÃO}

a - Pasmodex 0,25mg/ml, Isofarma. Eusébio - CE.

b - Anasedan injetável, Vetbrands. Jacareí - SP.

c - Cloridrato de tramadol injetável $50 \mathrm{mg} / \mathrm{ml}$, Cristal Pharma. Belo Horizonte - MG.

d - Ketamina Agener 10\% injetável, Agener União. Embu-guaçu - SP.

e - Cetoprofeno gotas, Medley. Campinas - SP.

\section{COMITÊ DE ÉTICA E BIOSSEGURANÇA}

A pesquisa desenvolvida foi previamente aprovada pela Comissão de ética para Uso de Animais (CEUA) da UFRPE, sob licença nº 026/2009.

\section{REFERÊNCIAS}

CHIORATTO, R.; TUDURY,E.A. Celiotomia - laparotomia. In: TUDURY, E.A.; POTIER, G.M.A. Tratado de técnica cirúrgica veterinária. São Paulo: MedVet, 2009. Cap.15, p.223-242.

CONCANNON, P.W.; MEYERS-WALLEN, V.N. Current and proposed methods for contraception and termination of pregnancy in dogs and cats. J Am Vet Med Assoc, v.198, n.7, p.1214-1225, 1991.

DORN, A.S.; SWIST, R.A. Complications of canine ovariohysterectomy. J Am Anim Hosp Assoc v.13, n.1, p.720$724,1977$.

ENDO, R.M. et al. Fármacos contraceptivos. Rev Cient Elet Med Vet, Ano VII, n.12, 2009. ISSN: 1679-7353.

ESMERALDO, B.A.M. et al. Benefícios e transtornos oriundos da administração exógena de contraceptivos progestacionais em felinos. VII Jornada de Ensino, Pesquisa e Extensão da UFRPE. Universidade Federal Rural de Pernambuco. Anais. Recife: JEPEX, 2009.

EVANS, H.E.; CHRISTENSEN, G.C. The urogenital system. In: EVANS, H.E. Miller's anatomy of the dog. 3. ed. Philadelphia: Saunders, 1993. Cap. 9, p. 531-546.

FIGUERÊDO, E.D. et al. Permeabilidade tubária e gestação após reversão de laqueadura. Rev Bras Prom Saúde. v.19, n.4, p.209-215, 2006.

JOHNSON, C.A. Distúrbios do ciclo estral. In: NELSON, R.W.; COUTO, C.G. Medicina interna de pequenos animais. $3^{\text {a }}$ ed. Rio de Janeiro: Elsevier, 2006a. Cap. 56, p.811-832.

JOHNSON, C.A. Pseudociese, distúrbios da prenhez, parto e período pós-parto. In: NELSON, R.W.; COUTO, C.G. Medicina interna de pequenos animais. 3 . ed. Rio de Janeiro: Elsevier, 2006b. Cap. 59, p.849-868. 
JONES, T.C. et al. Distúrbio do crescimento: Aplasia até neoplasia. In: Patologia veterinária. 6. ed. São Paulo: Manole, 2000. Cap. 4, p. 87-118.

MODOTTE, W.P. et al. Laqueadura tubária por microlaparoscopia sob anestesia local e sedação consciente. Rev Bras Videocir v.2, n.3, p.139-147, 2004.

MOORE, K.L.; PERSAUD, T.V.N. Malformações congênitas. In: Embriologia básica. 6. ed.. Rio de Janeiro: Elsevier, 2004. Cap. 9, p.122-143.

PIBOT, P. et al. Encyclopedia of Feline Clinical Nutrition. Centro de pesquisa e desenvolvimento da Royal Canin Paris: Aniwa Publishing, 2008. 509p.

REGGIANI, C.P.D. et al. Tubal sterilization and reversal: analysis of 21 cases. J bras Med. v.79, n.5, p.44-50, 2000.

RUDY, R.L. Stifle joint - joints of the hind limb. In: ARCHIBALD, J. Canine surgery. 2. ed. California: American Vet. Publications, 1974. Cap. 26, p.1156.
SANTOS, F.C. et al. Complicações da esterilização cirúrgica de fêmeas caninas e felinas. Revisão de literatura. Vet e Zootec. v.16, n.1, p.8-18, 2009.

SOARES, J.A.G.; SILVA, P.A.R. Castração precoce em cães e gatos. Clín Vet, Ano III, n.13, p.34-40, 1998.

STEELE, S.J. The potential for improved abdominal procedures and approaches for tubal occlusion. Int J Gynecol Obstet. v.51, n.1, p.17-22, 1995.

STONE, E.A. Reproductive system. In: SLATTER, D. Textbook of small animal surgery. 3. ed. Vol. 2. Philadelphia: Saunders, 2002, v.2, sec. 11, chapt. 98, p.1487-1502.

VIANA, F.A.B. Guia terapêutico veterinário. $2^{\mathrm{a}}$ ed. Lagoa Santa: Editora Cem, 2007. 463p.

WILSON, E.W. Sterilization. Baillieres Clin Obstet Gynaecol. v.10, n.1, p.103-119, 1996. 